\title{
Relationship between platelet count and hemodialysis membranes
}

This article was published in the following Dove Press journal: International Journal of Nephrology and Renovascular Disease 17 August 2013

Number of times this article has been viewed

\author{
Rabih Nasr' \\ Chadi Saifan' \\ Iskandar Barakat ${ }^{2}$ \\ Yorg Al Azzi ${ }^{2}$ \\ Ali Naboush ${ }^{2}$ \\ Marc Saad ${ }^{2}$ \\ Suzanne El Sayegh' \\ 'Department of Nephrology, Staten \\ Island University Hospital, Staten \\ Island, NY, USA; '2Department \\ of Medicine, Staten Island University \\ Hospital, Staten Island, NY, USA
}

\begin{abstract}
Background: One factor associated with poor outcomes in hemodialysis patients is exposure to a foreign membrane. Older membranes are very bioincompatible and increase complement activation, cause leukocytosis by activating circulating factors, which sequesters leukocytes in the lungs, and activates platelets. Recently, newer membranes have been developed that were designed to be more biocompatible. We tested if the different "optiflux" hemodialysis membranes had different effects on platelet levels.
\end{abstract}

Methods: Ninety-nine maintenance hemodialysis patients with no known systemic or hematologic diseases affecting their platelets had blood drawn immediately prior to, 90 minutes into, and immediately following their first hemodialysis session of the week. All patients were dialyzed using a Fresenius Medical Care Optiflux polysulfone membrane F160, F180, or F200 (polysulfone synthetic dialyzer membranes, $1.6 \mathrm{~m}^{2}, 1.8 \mathrm{~m}^{2}$, and $2.0 \mathrm{~m}^{2}$ surface area, respectively, electron beam sterilized). Platelet counts were measured from each sample by analysis using a CBC analyzer.

Results: The average age of the patients was 62.7 years; 36 were female and 63 were male. The mean platelet count pre, mid, and post dialysis was 193 (standard deviation \pm 74.86 ), 191 (standard deviation \pm 74.67 ), and 197 (standard deviation \pm 79.34 ) thousand $/ \mathrm{mm} 3$, respectively, with no statistical differences.

Conclusion: Newer membranes have no significant effect on platelet count. This suggests that they are, in fact, more biocompatible than their predecessors and may explain their association with increased survival.

Keywords: platelet count, polysulfone membranes, complement activation, electron beam sterilized, cellulosic membranes, bioincompatible events

\section{Background}

In most countries, hemodialysis (HD) remains the main renal replacement therapy. There are more than 1.7 million patients treated with HD at about 28,500 dialysis units worldwide. ${ }^{1}$

HD has always been associated with complications; however, in this era of enhanced technology, complications due to HD are few compared to other procedures. That being said, some of these complications are related to the bioincompatibility of the dialyzer membranes used. These bioincompatible events range from leukopenia to complement activation and thrombocytopenia and might lead to an increased tendency for bleeding. ${ }^{2,3}$ The biocompatibility of the dialyzer membrane determines the extent of complement activation. Conflicting results have been published in the literature regarding cellulosic and biosynthetic membranes and their effect on platelet count.
Correspondence: Rabih Nasr Staten Island University Hospital, 475 Seaview avenue, Staten Island, NY 10305, USA

Tel + I 7I82269000

Email nasr_rabih@yahoo.com 
James Post demonstrated transient thrombocytopenia associated with HD using the Fresenius medical care optiflux polysulfone membrane (F-160) which is considered a highly compatible membrane. ${ }^{4}$

According to previous studies, cellulosic membranes are considered highly incompatible as opposed to the more biocompatible synthetic membranes. ${ }^{1,2,5,6}$ To date, there have been no studies that address the biocompatibility and the preference of one membrane over another. This interaction between the HD membrane and the patient's blood could also lead to alternate complement activation which has been associated with neutropenia and thrombocytopenia. ${ }^{4}$ The biochemical characteristics of the HD membranes determine the extent of complement activation. Several free hydroxyl groups are attached to the cellulose or cuprophane membranes that could activate complement with the highest intensity. ${ }^{4}$

Feyisayo Olafiranye et al, in another case report, showed thrombocytopenia in an HD patient using the F200 NR polysulfone synthetic dialyzer who then recovered when switched to the cellulose triacetate membrane. ${ }^{7}$

In this study conducted at Staten Island University Hospital, we followed 99 patients on HD using the optiflux polysulfone membrane, which is a synthetic membrane and we determined if there is a drop in platelets mid and post HD.

\section{Methods}

Ninety-nine patients undergoing 4-hour HD procedures three times per week for $\geq 6$ months were selected for the study. Informed consents were obtained prior to the study. It was approved by the Institutional Review Board of Staten Island University Hospital. The blood samples were collected at the beginning of the week, mainly on Monday and Tuesday. They were analyzed using the CBC analyzer Sysmex XT-4000i (Sysmex Corporation of America, Lincolnshire, IL, USA). All blood samples were kept at ambient temperature. The main dialyzer membrane used for HD was the optiflux polysulfone membrane, which was the Fresenius F160, F180, and F200 (Fresenius Medical Care North America, Waltham, MA, USA).

Exclusion criteria were the following: hematological cancers, myelodysplastic syndromes, baseline thrombocytopenia, acute infections, idiopathic thrombocytopenic purpura/thrombotic thrombocytopenic purpura patients, human immunodeficiency virus, and liver disease.

\section{Sample collection}

After obtaining informed consent, blood was drawn prior to dialysis, 90 minutes into dialysis, and immediately after dialysis. Blood samples for platelet counts were drawn in ethylenediaminetetraacetic acid (EDTA) tubes and kept at ambient temperature. They were measured by using the CBC analyzer Sysmex XT-4000i (Sysmex Corporation of America).

Complement levels were measured if platelets dropped more than $50 \%$.

\section{Statistics}

Mean and standard deviation (SD) of the three groups were calculated. Analysis of variance (ANOVA) was used to test for statistically significant differences in the mean platelet count for the three different time points.

\section{Results}

In our study, we measured the platelet count before, 90 minutes into dialysis, and immediately post dialysis. The outcome of interest was the platelet count, defined as the difference in platelet count at the start of dialysis ( 0 minutes), mid dialysis (90 minutes), and post dialysis.

The average age of the patients was 62.65 years. A total of 99 patients participated in the study and were followed over time. Thirty-six patients were female and 63 were male. The main dialyzer used was the Fresenius polysulfone optiflux 160, 180, and 200, and the CA 110 (Fresenius Medical Care North America).

The mean pre dialysis count was $193,000.86$ per $\mathrm{mm}^{3}$ with a SD of 74.86, the meanmid dialysis count was 191,00.27 per $\mathrm{mm}^{3}$ with a SD of 74.67 and the mean post dialysis platelet count was $197,00.16$ per $\mathrm{mm}^{3}$ with a SD of 79.34 (Figures 1 and 2).

The count, sum, average, and variance of each group were calculated. The $P$-value was 0.86 , thus we have statistically significant evidence at $\alpha=0.05$ to show that there is no difference in mean platelet count between the three groups.

An analysis to assess whether there is an association between the dialyzer type and the platelet post dialysis count was also performed. The $P$-value for the test of significance for the slope was $P=0.155$, indicating that there is no statistically significant association between the dialyzer type and the platelet count.

A similar multiple linear regression analysis was done to assess the association between age and gender, considered simultaneously, on platelet count post dialysis. The $P$-values for the tests of significance for the regression coefficient associated with gender and age were $P=0.86$ and $P=0.36$, respectively. These $P$-values indicate that there is no statistically significant difference between gender and age and platelet count post dialysis (Figure 1). 


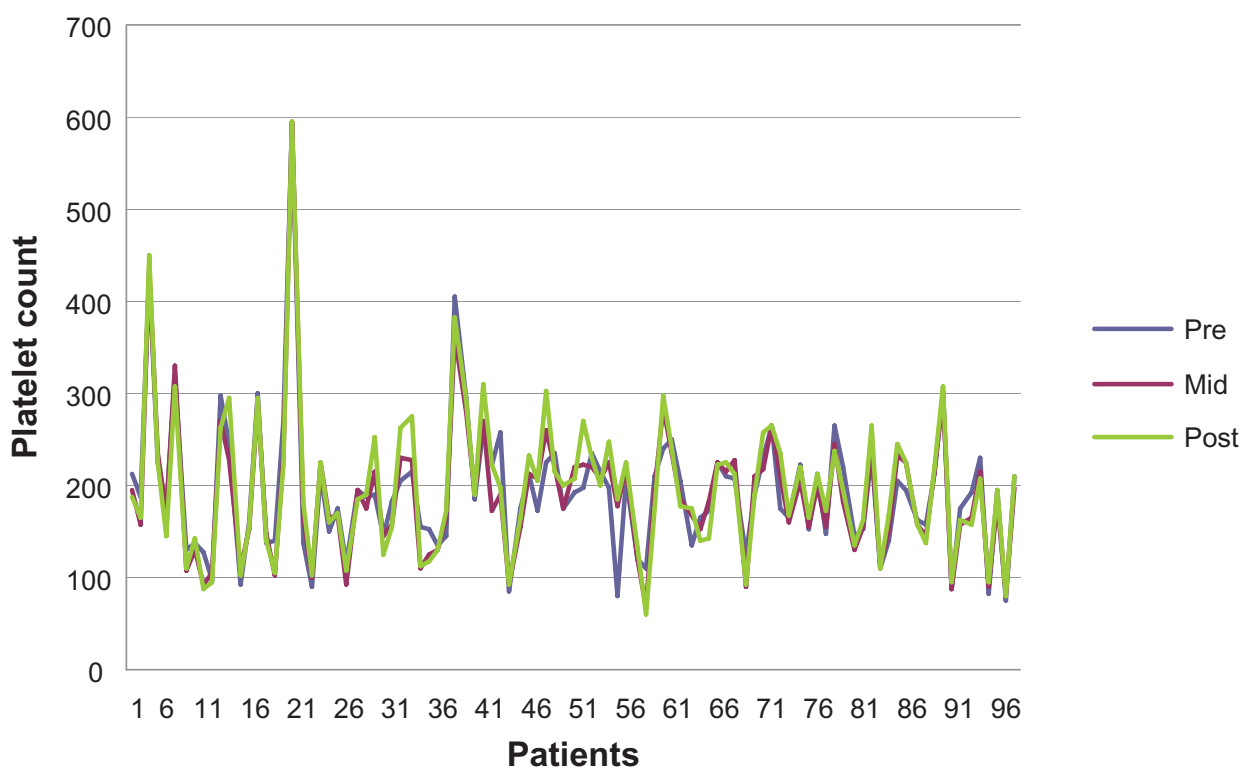

Figure I Platelet count pre, mid, and post dialysis.

\section{Discussion}

HD-associated thrombocytopenia has been described in patients undergoing HD with cellulosic (cuprophane) membranes. However, this event has rarely been described when using the highly biocompatible membranes, such as the optiflux membranes. There have been multiple reports describing HD-associated thrombocytopenia in patients undergoing HD with bioincompatible membranes, such as the cellulosic (cuprophane) membrane, and this has been clearly demonstrated by Schaffer and Hakim who reported HD-associated thrombocytopenia when using the cuprophane membrane, which did not occur when using the highly biocompatible, non complement activating dialyzer membrane, polymethylmethacrylate. There have been two case reports describing HD-associated thrombocytopenia when using the highly biocompatible synthetic membranes (optiflux F160,

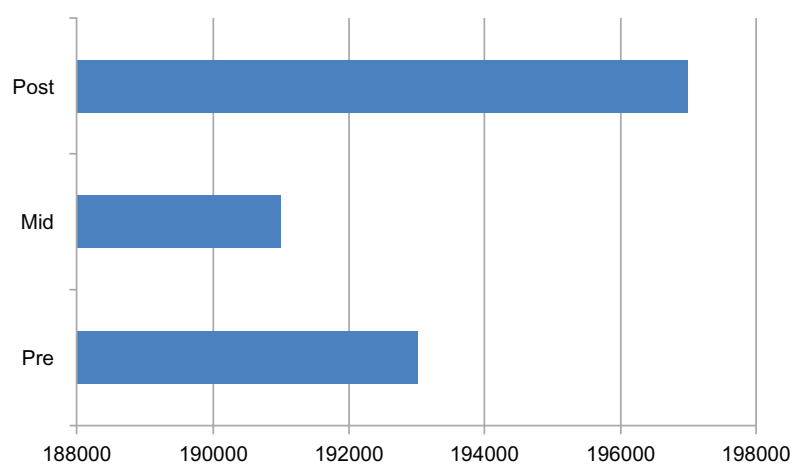

Figure 2 The platelet counts at the three different time points.

Notes: The mean pre dialysis count was 193,000.86 per $\mathrm{mm}^{3}$, mid dialysis count was $191,000.27$ per $\mathrm{mm}^{3}$, and the mean post dialysis platelet count was $197,000.16$ per $\mathrm{mm}^{3}$. optiflux F200 NR; Fresenius Medical Care North America) $)^{4,7}$ in patients undergoing hemodialysis.

In this study, we demonstrated no drop in platelet count 90 minutes through dialysis and post dialysis using the Fresenius optiflux membranes (F160, F180, F200 and the CA110; Fresenius Medical Care North America).

Biomaterials have been regularly used in different types of artificial tissues and organs, such as the plasmapheresis equipment, hemodialysers, catheters, and prostheses; biocompatibility has always been a concern with these devices. Several bioincompatible events have been described, such as in HD where the dialysis membranes can trigger whole body inflammation leading to accelerated arteriosclerosis. ${ }^{8}$

The contact of blood with the dialyzer membrane triggers an inflammatory reaction similar to what occurs during infection. Some of the proposed mechanisms are that this inflammatory reaction triggered by the dialyzer membrane leads to the activation of the coagulation cascade, which causes the release of activated Factor 10. This continuous state of activation might assist in the chronic inflammatory state which would lead to the several HD associated comorbidities. ${ }^{9}$ It is the membrane itself, along with the biochemical characteristics and composition, that determine the extent of complement activation.

Biocompatibility has been of greatest concern when using the cellulose membranes but it is of a much lesser concern when using synthetic membranes such as the polysulfone. According to previous studies, polysulfone membranes are considered highly biocompatible and complement activation occurs less frequently with these devices. The synthetic 
membranes are comprised of hydrophobic materials that have a low tendency to activate the complement. On the contrary, cellulosic membranes (cuprophane) activate the complement to the highest extent; these are made of polysaccharide units with hydroxyl groups that are responsible for complement activation and biochemical interactions. This was previously demonstrated by Schaffer and Hakim where transient and significant thrombocytopenia occurred with the use of cuprophane membranes.

HD-associated thrombocytopenia could be a contributing factor to the increased tendency for bleeding in HD patients. This was demonstrated in a report where massive gastrointestinal bleeding was associated with thrombocytopenia in HD patients that resolved when switching to another dialyzer. ${ }^{7}$

In another study done on ten HD patients, a drop in platelets was demonstrated when using the cellulose acetate membrane. ${ }^{2}$ Recently, one case report demonstrated significant transient thrombocytopenia with HD in a patient using the highly compatible Fresenius Medical care optiflux polysulfone membrane (F-160; Fresenius Medical Care North America). Thrombocytopenia significantly improved when switching the dialyzer to the Asahi REXEED 25S (AR-25S; Asahi Kasei Medical Co., Ltd., Tokyo, Japan), which is another highly biocompatible membrane. ${ }^{4}$ In another case report, a patient developed thrombocytopenia while on HD using the F200 NR polysulfone synthetic dialyzer (Fresenius Medical Care North America), and it resolved when switching to the cellulose triacetate membrane which is considered semisynthetic. ${ }^{7}$

In our study, we followed 99 patients on HD using the highly compatible Fresenius polysulfone optiflux F 160, 180, and 200, and the CA110 (Fresenius Medical Care North America). The platelet count was determined by drawing blood at the end of the dialysis session to compare the variation between the counts taken during three different time periods; there were no differences in the mean platelet count pre, mid and post dialysis. Patients were selected randomly who were on dialysis using the polysulfone optiflux membrane.

Analysis also showed that there were no differences in the mean platelet count before, mid, and post dialysis between the optiflux 160, 180, 200, and CA110 (Fresenius Medical Care North America). Therefore, results could not have been affected by the type of the optiflux membrane. $P$-values were not significant regarding the age and gender and platelet count post dialysis. This demonstrates that age and gender does not affect the results of platelets post dialysis.
This study was an observational study and, therefore, no cause effect could be concluded. Also, the number of subjects was limited. More studies are needed with a higher number of participants. In the recent article by Kiaii et al, they showed thrombocytopenia occurring in dialyzers that were sterilized using the electron beam sterilization technique. ${ }^{10}$ This was not observed in our study in which all the dialyzers were electron beam sterilized.

\section{Conclusion}

In this study, we evaluated the change in platelet count in patients undergoing HD using the highly biocompatible electron beam sterilized membrane optiflux and we demonstrated no drop in platelets. In previous case reports, HD-associated thrombocytopenia was reported when using the optiflux membrane.

In conclusion, platelet count did not drop mid and post dialysis when using the polysulfone optiflux membrane.

\section{Disclosure}

The authors report no conflicts of interest in this work.

\section{References}

1. Floege J, Johnson RJ, Feehally J. Comprehensive Clinical Nephrology, 4th ed. St. Louis, MO: Elsevier; 2010.

2. Verbeelen D, Jochmans K, Herman AG, Van der Niepen P, Sennesael J, De Waele M. Evaluation of platelets and hemostasis during hemodialysis with six different membranes. Nephron. 1991;59(4):567-572.

3. Vicks SL, Gross ML, Schmitt GW. Massive hemorrhage due to hemodialysis-associated thrombocytopenia. Am J Nephrol. 1983;3(1):30-33.

4. Post JB. Thrombocytopenia associated with use of a biocompatible hemodialysis membrane: a case report. Am J Kidney Dis. 2010;55(6): e25-e28.

5. Raymond MH, Schutte BC, Torner JC, Burns TL, Willing MC. Osteocalcin: genetic and physical mapping of the human gene BGLAP and its potential role in postmenopausal osteoporosis. Genomics. 1999;60(2):210-217.

6. Mwaniki DL, Courtney JM, Forbes CD, Paul JP. Assessment of blood compatibility of haemodialysis membranes using a miniature flat sheet dialyser. East Afr Med J. 1992;69(3):149-152.

7. Olafiranye F, Kyaw W, Olafiranye O. Resolution of dialyzer membraneassociated thrombocytopenia with use of cellulose triacetate membrane: a case report. Case Rep Med. 2011;2011:134295.

8. Nilsson B, Ekdahl KN, Mollnes TE, Lambris JD. The role of complement in biomaterial-induced inflammation. Mol Immunol. 2007;44(1-3): 82-94.

9. Pertosa G, Simone S, Soccio M, et al. Coagulation cascade activation causes CC chemokine receptor-2 gene expression and mononuclear cell activation in hemodialysis patients. JAm Soc Nephrol. 2005;16(8): 2477-2486.

10. Kiaii M, Djurdjev O, Farah M, Levin A, Jung B, MacRae J. Use of electron-beam sterilized hemodialysis membranes and risk of thrombocytopenia. JAMA. 2011;306(15):1679-1687. 


\section{Publish your work in this journal}

The International Journal of Nephrology and Renovascular Disease is an international, peer-reviewed open-access journal focusing on the pathophysiology of the kidney and vascular supply. Epidemiology, screening, diagnosis, and treatment interventions are covered as well as basic science, biochemical and immunological studies. The journal welcomes original research, clinical studies, reviews \& evaluations, expert opinion and commentary, case reports and extended reports. The manuscript management system is completely online and includes a very quick and fair peerreview system, which is all easy to use. Visit http://www.dovepress.com/ testimonials.php to read real quotes from published authors

Submit your manuscript here: http://www.dovepress.com/international-journal-of-nephrology-and-renovascular-disease-journal 